\title{
HUMAN-ROBOT COEXISTENCE IN ROBOT-AIDED APARTMENT
}

\author{
R. Correal, A. Jardón, S. Martínez, R. Cabás, A. Giménez, C. Balaguer \\ Robotics Lab \\ Department of System Engineering and Automation \\ University Carlos III of Madrid, Spain \\ c/ Butarque, 15, 28911, Leganés (Madrid), Spain \\ http://roboticslab.uc3m.es/ \\ [rcorreal, ajardon, scasa, rcabas, agimenez, balaguer]@ing.uc3m.es
}

\begin{abstract}
Historically the kind of technologies used in homes has been in the form of electrical appliances such as washing machines, ovens, etc. Later was introduced the classic "Home Automation" concept, which involves improvements like computer-controlled devices such as lights, alarms, different sensors, etc. The classic devices have always been static, this means they are installed in the house and stay there during all their useful life doing always the same task. In this paper is presented the next step in the introduction of advanced technologies in home environments. The main concept is to introduce dynamic and mobile items in homes, it means robots. The developed robot ASIBOT is a mobile device, different to classic mobile robots, which can travel around the house and can help to perform a wide variety of task: eating, cooking, washing, transportation, etc. helping people wherever it would be needed.
\end{abstract}

Keywords: Robotics, Ambient Intelligent, Assistive, Disabled, Home Automation.

\section{INTRODUCTION}

New devices are introduced everyday in homes to make them more comfortable and useful through the automation of many daily life aspects. Ambient Intelligence builds on supported by TICs. Concrete Ubiquitous Computing integration of low cost microprocessors into everyday objects like furniture, clothing, white goods, walls, etc. all these processors are able to talk with the others and Intelligent User Interfaces, that allows the user to be integrated into the ad-hoc wireless communication systems. The AmI concept offers and excellent opportunity to, not only the improvement of domestic environments towards a more comfortable ones, but also to introduce a set of new technology systems specially useful for elderly and people with special needs achieving a better way of life and reach a higher level of independence.

Historically the kind of technologies used in homes has been in the form of brown and white lines, e.d., electrical appliances such as washing machines, ovens, etc. Later was introduced the classic "Home Automation" concept, which involves the introduction of sensors, allowing to achieve improvements based on low cost microprocessorcontrolled devices such as lighting and temperature control, intrusion detection, fire alarms,, etc. But this classic devices have always been static, this means they are installed in the house and stay there during all their useful life doing always the same task. In order to improve the ratio profit/ cost several strategies are revised, [1]

This paper presents the next step in the introduction of advanced technologies in home environments, according with the concept of "modular autonomy" introduced by
P.Dario [2]. The main concept is to introduce dynamic and mobile items in homes, it means robots. Even though, this idea is not new [3],[4],[5],[6], previous attempts failed in achieve the desired functionality for several reasons: complex intelligence needed on board of the robot makes it huge expensive, not enough flexible to be used for many users or oversized functionalities that don't fit the users expectations. The developed robot ASIBOT [7] is a mobile device which can travel around the house and perform a wide variety of task: eating, cooking, washing, transportation, etc. helping people wherever it would be needed. In comparison with the classic electrical appliances, this robot is an assistant manipulator designed to help people. The robot is not fixed in a concrete location, it can be "working" in any place within the house. It can perform lots of different tasks -not just one, because is designed to be flexible and versatile- in any part of the house, at any time and it could be adapted to the concrete needs of each person.

There have been carried out several experiments in real environment with real disabled people in the National Hospital of Paraplegics in Toledo, Spain, where a complete apartment has been adapted and tested.

Currently a robot-aided kitchen is being developed at the Robotics Lab of the UC3M, keeping in mind the concept of "Design for All". The main goal is to check the humanrobot coexistence in a real kitchen: introduce the robot in it and teach it how to prepare a meal, establishing communication with different devices within the kitchen and house in order to use and control them, such a microwave oven, electric cooker, etc. Also the security 
issues of the human-robot coexistence will be analyzed in order to guarantee the " $100 \%$ " security.

\section{AMBIENT INTELLIGENT}

As described in the book Ambient Intelligent from Emerging Communication [8]: “Ambient Intelligence implies a seamless environment of computing, advanced networking technology and specific interfaces. It is aware of the specific characteristics of human presence and personalities, takes care of needs and is capable of responding intelligently to spoken or gestured indications of desire, and even can engage in intelligent dialogue. Ambient Intelligence should also be unobtrusive, often invisible: everywhere and yet in our consciousness nowhere unless we need it. Interaction should be relaxing and enjoyable for the citizen, and not involve a steep learning curve."

With ambient intelligent we understand a new concept of the classic Home Automation, the next step in the introduction of technology in home environments. As AmI is a technology intrinsically designed for all, it will be the low cost and effective way to make the robotics assistant reliable, useful, and autonomous in close interaction with the smart environment [9]. In the context of this development a mobile robot is introduced within the environment in the most unobtrusive way possible, the robot will use the walls to move from one point to another. In most cases the walls are underutilized, that way the floor remains free, in order to let the users, probably using a wheelchair, moving easier around the environment.

The idea is to use the robot not just as an assistive helper, but as a kind of "butler". This means the robot will be another device the user will use. The interaction with the robot is supported by intelligent HMI. That way, the rest of the elements are controlled through the HMI, which allows multimodal interaction in order to let the users to give commands to the Smart Environment, using one of the multiple ways to interact with the robot like tactile, a joystick or human voice recognition. Through using these devices a user could, for instance in the kitchen, open and start an oven or microwave, check the foods stocked at the fridge control the electric cooker or send a purchase order to the grocery store.

\section{ASSISTIVE ROBOT ASIBOT}

The ASIBOT robot has five degrees of freedom, and it is divided in two parts: the tips that have a docking mechanism (DS) to connect the robot to the wall or a wheelchair, and a gripper. The body has two links that contain the electronic equipment and the control unit of the arm. In this way the robot is self-constrained being portable with overall weight of $11 \mathrm{Kg}$. It is important to note that the robot is symmetric, due to it is possible to fix the arm in any of its ends. The raw material is made by aluminium and carbon fibber. The actuators are torque DC motors, and the used gears are flat Harmonic-Drive. The power supply is taken from the connector that is placed in the centre of the docking station. The range and the position of the different joints can be seen in Fig. 1.

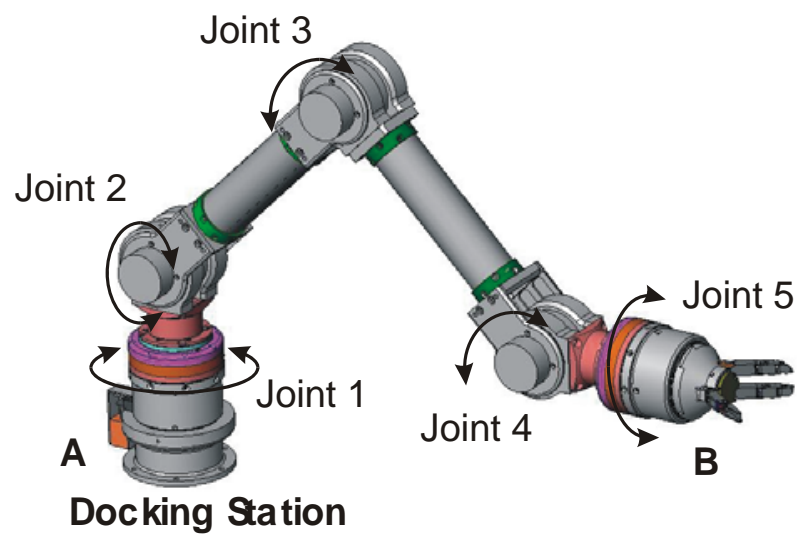

Fig.1. ASIBOT robot design and its degrees of freedom

ASIBOT is designed to be modular and capable of fitting into any environment. This means that the robot can move accurately and reliably between rooms and up or downstairs, and can transfer from/to the wheelchair [10]. For this purpose the environment is equipped with serial docking stations which make possible the transition of the robot form one of them to another one. This degree of flexibility has significant implications for the care of the disabled and elderly people with special needs. The modularity of the system makes possible the system grows as the level of disability of the user changes.

The main functions of the DS are: to fix mechanically the tip of the robot that it's working as a base; and also provide $24 \mathrm{~V}$ power supply. Three different kinds of DS (fig, 2) have been developed: a) Fixed DS. These kinds of mechanisms are fixed to the walls, ceils, furniture and other places of the house where it is needed for each task such as in the table for placing the plates into the dishwasher. b) Mobile DS. When the robot needs to move a long distance between two DS it is better to move in high velocity. This is possible if the DS can move in a rail into the wall or table. c) DS on the wheelchair. It is a special DS, located on a rail mounted in the wheelchair. There are special DS in any room from which the transition between a fixed DS and the wheelchair is allowed.

\subsection{ASIBOT Robot applications}

The main applications of the robot are involved in domestic tasks. It is not necessary a high degree of precision during these motions, less than when the robot is moving between two DS. During the design process it was decided that while performing eating and shaving tasks the only action that the robot should do is to present the spoon, the shaver, or the toothbrush to the user. Fig. 3 shows several working environments where ASIBOT robot cooperates to perform some domestic tasks, such as 
shaving and drinking. During these tasks it is very important the control of the different trajectories of the arm and its velocity and acceleration profile, because the robot will move very close to the user. If the robot is moving a spoon with meal, it will be crucial the control of the orientation of its extreme in order to avoid throwing down the meal.

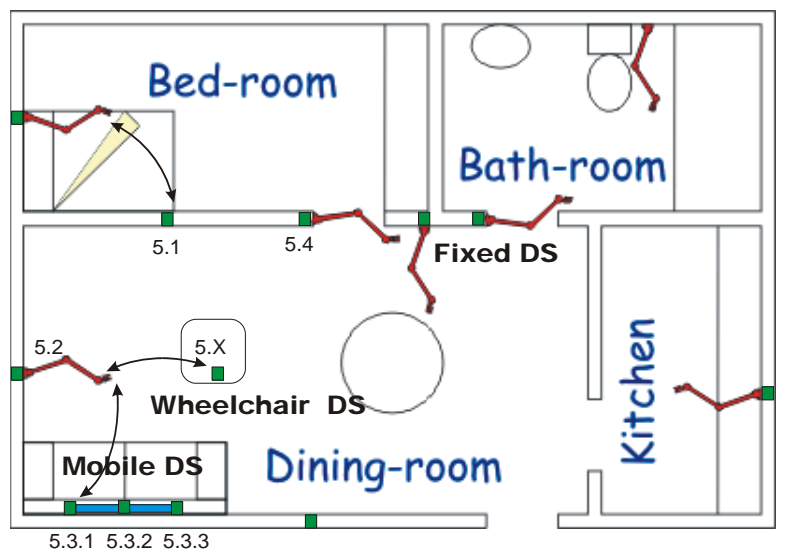

Fig.2. Concept of robot-aided apartment

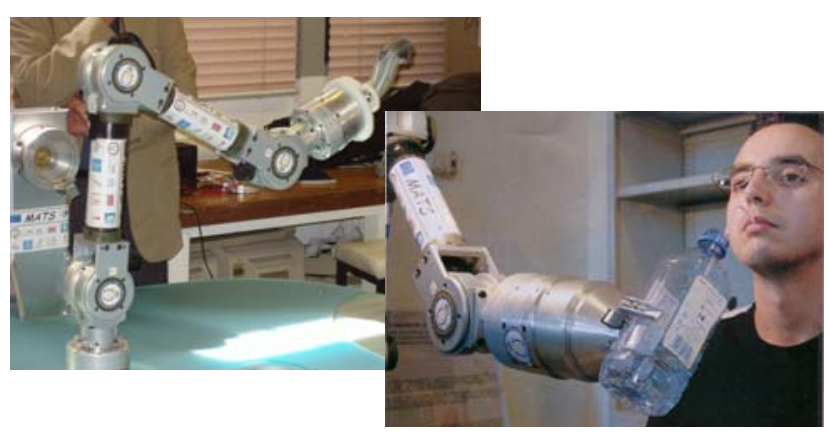

Fig.3. The ASIBOT robot connected to two different fixed docking stations during a shaving and drinking tasks

\subsection{Control architecture}

Fig. 4 shows the overall control architecture of the ASIBOT system. Three different levels of computational tasks are considered, and that are implemented in the following subsystems:

1. The Human Machine Interface (HMI)

2. The Room Controller (RC)

3. The Arm Controller (AC)

The HMI is the device available to the user: a) to command the arm functionality; b) to be informed about the state of the device or the task the robot is involved; c) to benefit from the navigational feedback offered during the transfer manoeuvres from the wall-mounted docking station to the wheelchair docking station and vice-versa; d) to get access to standard application software, including Internet browser and e-mail.

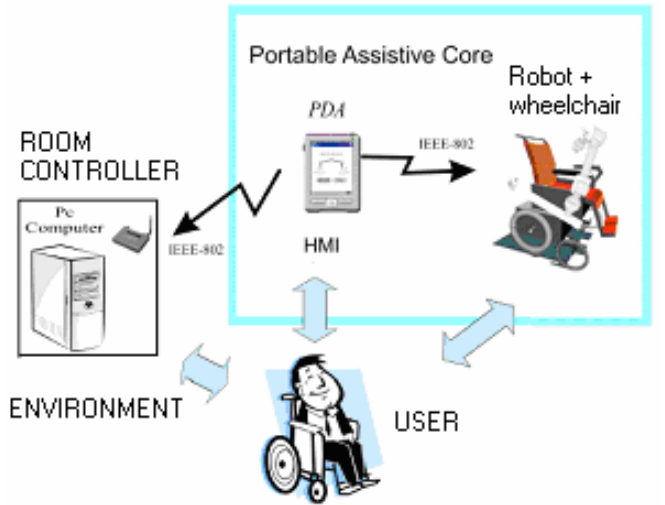

Fig.4. Overall architecture of the assistive system

The RC is a computer whose main functions are: a) to perform path-planning activities, in order to the arm be optimally moved within the network of available docking stations from a given start point to a specified target configuration; b) to select and send the list of motion commands needed by the AC to move the robot arm; c) to perform wheelchair localisation via a monocular visionbased system built around a web-cam image sensors (sensor-based assistance to docking for management of arm transfer procedures) [11].

The AC is embedded within the robot arm structure. The main functions of the AC are: a) communication management (to interact with the clients, PDA or RC) b) commands interpreter, c) kinematics transformations (direct kinematics and inverse kinematics), d) pathplanning (for straight line movements), e) connection to the amplifiers, and f) commands to digital inputs and outputs, (i.e.: to open and close the grippers).

\section{HUMAN FACTORS OF DISABLED USERS}

The key part in the control architecture of any assistive robot is the usability of the HMI, because the overall performance is HMI dependant. Interaction devices addresses several mutually exclusive design trade-offs and complications. Users, by the nature of their potential benefit from an assistive robotic device, are also very limited in the ways in which they can interact with the device. Simultaneously, device specification is variable. However, direct control is good to avoid uncertainly but task execution is tedious. Executing a pre-programmed task is much faster, yet such systems cannot meet some of the user's requirements and the effort required to program a task has been criticized. A need has been marked for a non-technically oriented person to be provided with easy tools for performing task or programming tasks [11] [4]. The conflicting constraints are to maximize flexibility while minimizing the length of time it takes to perform a task and minimizing the cognitive load placed on the user

In order to design an interface for an assistive robot which allows the user to be 'in the loop' as the main part of the 
interaction architecture, the ASIBOT robot takes into account considerations like: portability of the HMI and device dimensions, flexibility and connectivity, versatility, high degree us usability for non skilled users, reduce the mental load to the user, updatability and expandability -in order to connect new peripheral devices- and scalability.

\subsection{ASIBOT HMI}

Every group of users has different characteristics, abilities and possibilities, but most of them are expected to have mobility problems and probably need to use a wheelchair.

The device selected to implement the user interface is a PDA (Pocket PC) for many reasons. One of them is the small size and weight this kind of devices have. This allows to be carried out easily by any user or to be attached to a wheelchair in a place visible to the user. It also has very low power consumption. Another characteristic is the easy and versatile ways of control that a PDA allows. The front of the device is a screen, which offers tactile capabilities. It almost doesn't have buttons, it is just a screen (fig. 5).

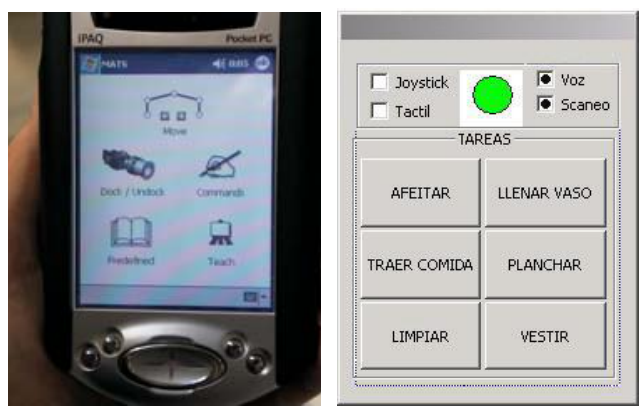

Fig.5. PDA-based users interfaces of the ASIBOT robot

There have been developed different ways to control the robot using a PDA. These possibilities are: tactile screen, using a pointer or a finger, using a scanning system and a button to select options, attaching a joystick and a voice recognition system. There is also possible to combine some of these control modes, in order to adapt the interface as much as possible to the concrete needs of every user.

\section{ROBOT'S USER ACCEPTANCE}

The motivation for user trials is the interest to seek directly the potential disabled user's opinion about the use of the robotic aid in their homes and workplaces. We try to focused in the detection of acceptance level, identify prejudices and fears, uncovered needs and expectations [12].

The protocol followed was applied with two different scenarios. The first, live demos at laboratory with user displaced from rehabilitation centres, and second, demos with patients far away in communication with the users that control the robot via teleconference. In both cases the demo was divided in two stages:
Six scenarios or tasks have been selected for user evaluation assisted by the ASIBOT: eat, drink, shave, make-up, pick and place objects, and arm operating from a wheelchair. A brief explanatory report of the system was kicked to the user. Information was collected by an examiner via an open and close-ended questionnaire. Results obtained must be correlated with the nature of the user's pathologies, culture, residuals motor abilities, etc.

After data gathering and analysis some results was generated, between those remarks user contributions of how to improve the system functionalities. Sometimes those proposals are contradictory and other seems to be closer to fiction than reality, for example, reduce the size and at the same time increase the distance between docking stations. The next are the main reasons for a low acceptability: too large, lack of doing things, risk of isolation, reduction of communication, bad appearance, frightening, too slow, too remote from my life.

Overall the subject group responded positively to the demonstration. They felt that the robot could make a welcome difference to their lives. Of the additional comments received $89 \%$ were positive. It was of concern that not being able to actually use the robot would mean that the subjects would have difficulty relating the robot to their real, everyday situation. This does not appear to be the case because whilst some were not able to relate it to their situation the majority felt that they could. When asked to express free ideas, the most popular tasks identified, were food preparation, household, and grasping high and low objects.

Slightly more than half the subjects felt that the robot would have an effect on the level of care/help they would need. Only $9.5 \%$ felt negative about this effect.

The size of the robot was thought to be the most significant factor. Further work is needed to understand exactly how changes to physical size would influence this. Time constraints for this final user evaluation have resulted in the condition profile of the subject sample being biased towards spinal injury (75\% spinal injury). This population is more likely to be driven towards greater levels independence. This could account for the relatively large number who felt that a reduction in care levels was positive. This in itself is an important result but more work is needed before generalisation across a wider spectrum of condition is possible.

The most positive tasks (ranked interesting or above): wheelchair transfer / gripping and releasing objects - over $75 \%$, drinking - $65 \%$, the largest area thought to be definitely not of interest was eating (approx 30\%). The physical size and speed of movement of the robot are likely to have had an effect on this result.

This illustrates the complex nature of evaluating this type of equipment and points towards the importance of a more experiential evaluation than has been possible at this time. 
There was significant support for some measure of direct control of the robot (as well as with pre-programmed), the use of a joystick / chin control and the voice recognition system were the most popular. The remote subjects were not able to perceive any possible difficulties of directing the end effector in 3 dimensional space from a two dimensional system such as a joystick.

As a final conclusion for these experimentation results it can be concluded that this system is more suitable for the most severe disabled people, since the ones with certain mobility in their arms prefer to do the tasks by themselves, even if they have to use adapted tools, therefore they don't need the help of this assistive system.

\section{EXPERIMENTAL RESULTS AT THE ROBOT AIDED-APARTMENT}

The above described features of this system contribute to the robotics and Ambient Intelligent research with a new concept of robotic aided-environment.

Has been introduced the concept of dynamic elements robots- collaborating with the environment and the user to create an intelligent ambient.

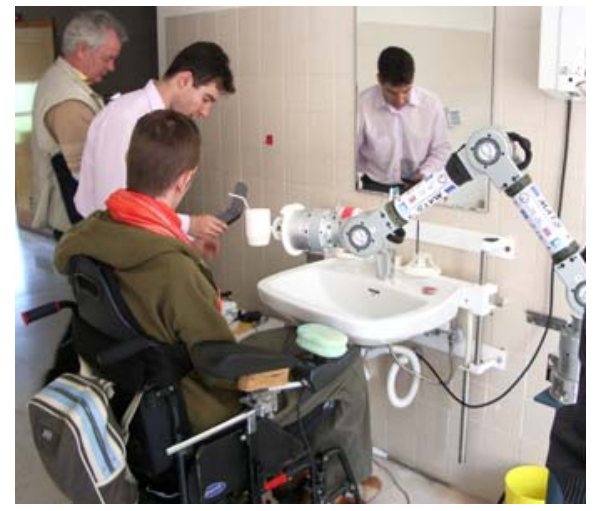

Fig.6. The robot in an adapted bath room at the Hospital

To test the system, an environment has been adapted in the National Hospital of Paraplegics of Toledo (Spain), see figure 6, where real disabled users are at the moment using and evaluating it. At the moment the robot has been tested in the living room environment, helping to perform tasks like eating, drinking, grasping objects, etc. and in the bathroom, assisting users to teeth-washing, make-up, etc. Fig. 7 shows the robot helping a user to eat.

The next step will be to extend that adaptation to the rest of the domestic environment, adapting a real kitchen. The final objective is to let the robot to complete the whole process of preparing by itself a simple meal. A mid-term main objective is the development of a second prototype much more intelligent, robust, safe [13], [14], [15] and user-friendly.

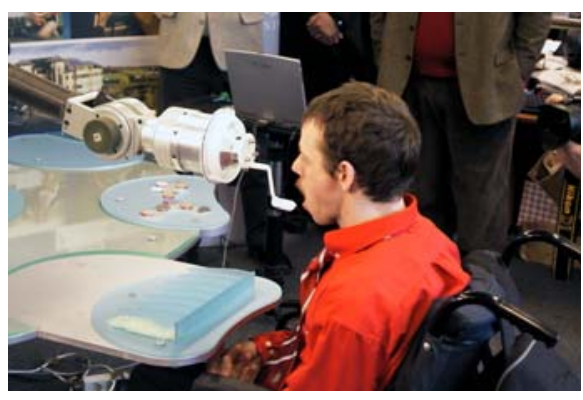

Fig.7. Eating task assistance by ASIBOT

The next picture shows prospective designs for such a kitchen, which is a normal one with little adaptations.
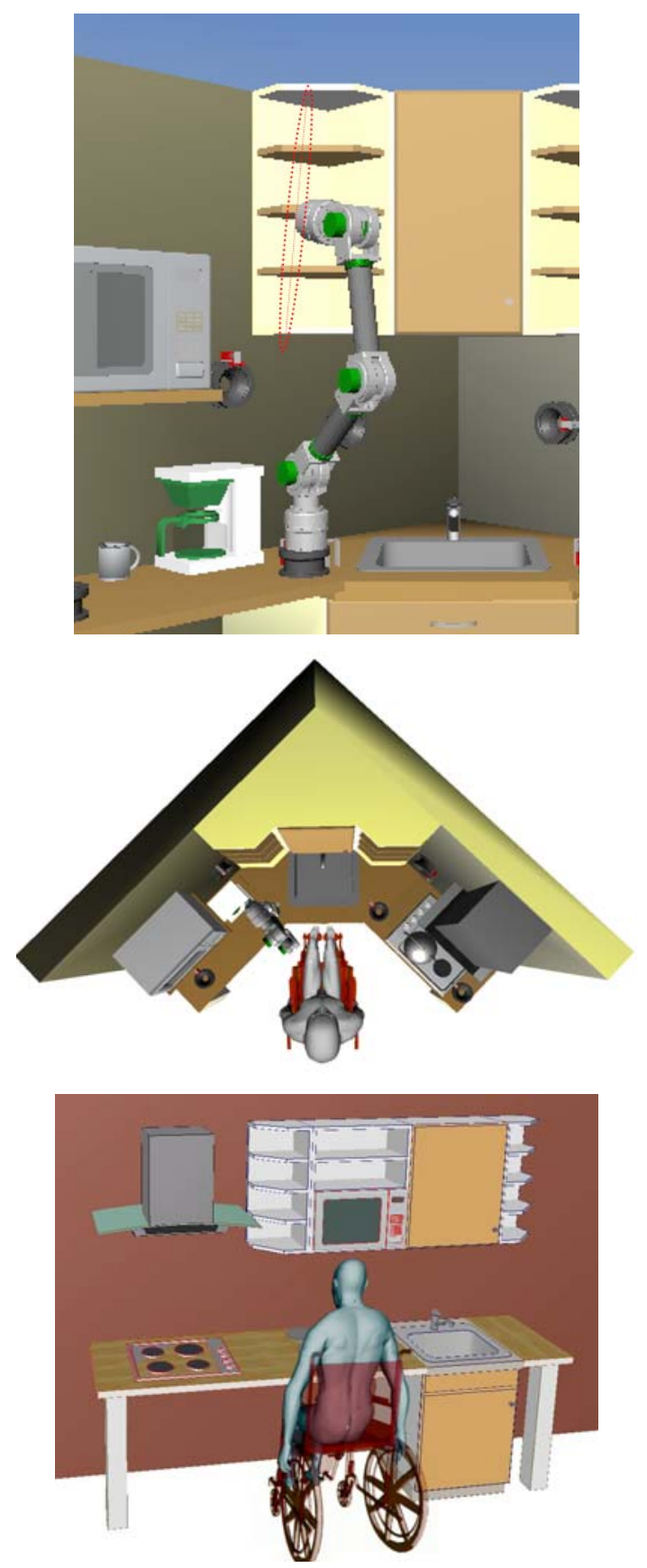


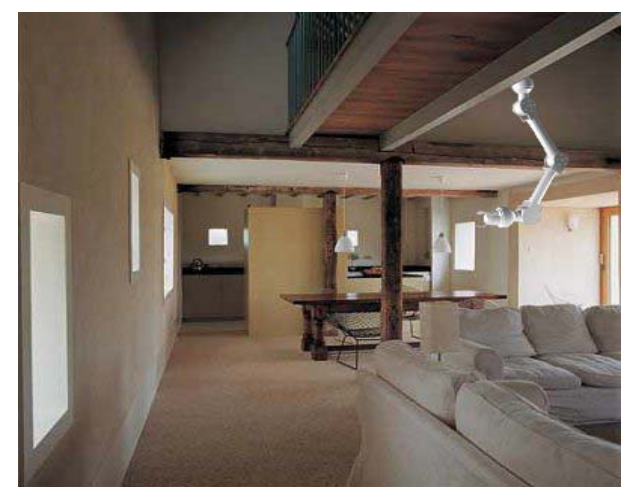

Fig.8. a) b) corner design of a kitchen, c) straight line design, d) the robot in the living room.

Depending on the level of disability of the user different types of HMI has been presented. Human factors are not only important for the commanding itself but also are crucial for security issues. The actual tests have demonstrated the feasibility of the system. During the first trials there was a good acceptance of the end-users.

The adaptation of the environment to let the system works is simple. It just requires to install the connectors (DS) in the right place in order to let the robot move around the house, minimizing the number of them and maximizing the reach.

Using the Yoshikawa [16] definition of manipulability, the best robot joints configuration is achieved for each task. The ellipsoids of manipulability obtained from the eigenvalues of the Jacobian matrix, allow to decide which are the better locations to fix each DS. The goals are to achieve the maximum manipulability, but in order to get the cheaper solution is necessary to minimize the number of DS, is a sub-optimal problem. Furthermore, sometimes the mathematical formulation give impossible solutions due to the physical constrains in the mechanical fixation of the DS.

\section{ACKNOWLEDGEMENT}

MATS is an European Union funded project IST 200132080, which was the precursor of this project. Thanks to all the MATS partners and to the RoboticsLab, at UC3M, research team.

ASIBOT is a project funded by the IMSERSO, within the Ministry of Labour and Social Affairs, by the National Plan of Scientific Research, Development and Technological Innovation (2004-2007).

\section{REFERENCES}

[1] C. Laschi, E. Guglielmelli, G. Teti, P. Dario, “A modular approach to rehabilitation robotics", in 2nd EUREL Workshop on Medical Robotics, Pisa, Italy, September 23-24, 1999, pp.85-89.
[2] Paolo Dario, Eugenio Guglielmelli, Cecilia Laschi, Humanoids and Personal Robots: Design and Experiments Journal of Robotic Systems 18(12), 673-690 (2001).

[3] Tou, an Assistant Arm: Design, Control and Performance». A. Casals. 6th Int. Conference on Advanced Robotics, Tokio-1993.

[4] [Eftring] H. Eftring, "Robot control methods and results from user trials on the RAID workstation", International Conference on Rehabilitation Robotics, 1994. [5] [Van der Loos et al.., 89] M.Van der Loos et al. Design and Evaluation of a Vocational Desktop Robot. Proc. RESNA 12th Annual Conf., New Orleans, 1989.

[6] M.Hillman: Rehabilitation robotics from past to present, a historical perspective,Bath Institute of Medical Engineering, Proceedings of the ICORR 2003, 23-25 April 2003.

[7] A.Giménez; A.Jardón; R.Correal; R.Cabas; C.Balaguer. "A portable light-weight climbing robot for personal assistance applications”, 8th International Conference on Climbing and Walking Robots (Clawar'05). London. UK. Sep, 2005.

[8] G. Riva, F. Vatalaro, F. Davide, M. Alcañiz. "Ambient Intelligence: The Evolution Of Technology, Communication And Cognition Towards The Future Of Human-Computer Interaction". Emerging Communication. OCSL Press, 2005.

[9] J. Crisman, G. Bekey, "Grand Challenges for Robotics and Automation: the 1996 ICRA Panel Discussion”, IEEE Robotics and Automation Magazine, Dec. 1996, pp.10-16. [10] C.Balaguer; A.Giménez; A.Jardón. "Climbing Robots Mobility for Inspection and Maintenance of 3D complex Environments”, Autonomous Robots. Vol. 18. No. 3. pp.157-169. 2005.

[11] A.M. Sabatini, V. Genovese, E.S. Maini: "BeViewer: vision-based navigation system to assist motor-impairred people in docking their mobility aids", IEEE International Conference on Roboitcs and Automation, ICRA 2003, Taipei, Taiwan.

[12] C.Balaguer; A.Giménez; A.Jardón; R.Cabas; R.Correal. "Live experimentation of the service robot applications elderly people care in home environments", IEEE/RSJ. International Conference on Intelligent Robots and Systems (IROS'2005). Edmonton. Canada. Aug, 2005. [13] M. Zinn, O. Khatib, B. Roth, and J.K. Salisbury, A new actuation approach for human friendly robot design, en Proc. Int. Symp. Experimental Robotics- ISER'02, Sant'Angelo d'Ischia, Italy, 2002.

[14] H.F.M. Van Der Loos, D.S. Lees, L.J. Leifer. “Safety considerations for rehabilitative and human service robot systems”, RESNA 15th Annual Conference,322-324, 1992. [15] Bicchi, A., Rizzini, L., and Tonietti, G. Compliant design for intrinsic safety: general issues and preliminary design. Proceedings of the International Conference on Intelligent Robots and Systems. 2001.

[16] Yoshikawa, Tsuneo. Foundations of robotics: analysis and control Editor: MIT Pres, Pags: 285 p. ISBN: 0262240289. 1990. 